\title{
Improving Tools and Options for the Management of Patients with Neuroendocrine Tumors: A Patient Perspective
}

\author{
Josh Mailman \\ NorCal CarciNET Community
}

See the associated article on page 1452.

$\mathbf{I}$ has been nearly 9 y since I first saw a presentation on ${ }^{68} \mathrm{Ga}$ DOTATOC PET/CT given by a European physician at a patient education conference. I had just been diagnosed with a rare neuroendocrine tumor and did not have many therapeutic options. I vividly remember Dr. Richard Baum from Germany addressing the audience on the third day of the conference after $2 \mathrm{~d}$ of lectures on surgery and long-acting somatostatin analog therapy, wondering how treatments were selected when the disease could not be accurately localized. Dr. Baum proceeded to talk about the benefits of imaging with ${ }^{68} \mathrm{Ga}$ PET/CT. I knew immediately that I would undergo this imaging procedure to have the clearest picture of the state of my disease. Little did I know that flying to Germany for a ${ }^{68} \mathrm{Ga}$-DOTATOC PET/CT in December 2008 would change the path of my disease management as well as my involvement in the nuclear medicine field. It is with this background that I am honored to write an introduction to the article by Graham et al. (1) that appears in this issue of The Journal of Nuclear Medicine.

As the authors pointed out, the standard of care for neuroendocrine tumor (NET) whole-body imaging had been the OctreoScan, first approved in June 1994. At the time of my NET diagnosis in 2007, it was the last diagnostic or therapeutic agent approved for the treatment of NETs. The authors also stated that NETs are a rare disease and although the incidence and prevalence continue to increase as reported by Dasari et al. (2), the standard of care has not progressed in several decades.

From a patient perspective, the OctreoScan is less than ideal. The entire process can take $2-3 \mathrm{~d}$ and may require use of a laxative (3). Because of the complexity of the scan and the resulting image quality, many treating physicians order only a single OctreoScan at the onset of diagnosis. The only perceived value of the OctreoScan was either to determine whether the disease was somatostatin subtype 2 receptor-positive for long-acting octreotide therapy or to see whether the disease had spread to regions not imaged by CT or MRI.

At the time of my first ${ }^{68} \mathrm{Ga}$-DOTATOC PET/CT in 2008, many U.S.-based treatment providers were skeptical of the need for an

Received May 2, 2017; revision accepted Jun. 26, 2017.

For correspondence or reprints contact: Josh Mailman (e-mail: Josh@ NorCalCarciNET.org).

Published online Jun. 29, 2017.

COPYRIGHT @ 2017 by the Society of Nuclear Medicine and Molecular Imaging. DOI: 10.2967/jnumed.117.191833 additional imaging method for NETs. Although Graham et al. show that ${ }^{68} \mathrm{Ga}$-DOTATOC PET/CT is an imaging method superior to the OctreoScan, many providers in 2008 felt it would not result in a change of management given the therapeutic treatment options available. I was personally convinced that ${ }^{68} \mathrm{Ga}$ DOTATOC $\mathrm{PET} / \mathrm{CT}$ should continue to be studied. In addition to more precise imaging, it is a more convenient procedure for the patient, requiring a single half-day visit to an imaging center and no use of a laxative.

My initial ${ }^{68} \mathrm{Ga}$-DOTATOC PET/CT showed no additional disease and no change in my treatment course because my disease was stable. In $3 \mathrm{mo}$, this would change as I developed symptoms that, while moderately controlled by somatostatin analogs, needed to be addressed with additional therapeutic options for control of my progression. With no approved treatment for my specific condition and no clinical trials available in the United States for which I was eligible, I again turned to the facility that had imaged me with ${ }^{68} \mathrm{Ga}$-DOTATOC PET/CT. The facility had been performing therapy for $10 \mathrm{y}$ using the same DOTATOC that I had been imaged with. In 2009-2010, I underwent 3 successful treatments of peptide receptor radionuclide therapy using DOTATOC as a diagnostic and therapeutic pair. As Dr. Graham demonstrates, my personal experience of a change in treatment was not unique. Centers around the world using ${ }^{68} \mathrm{Ga}$-DOTA agents were reporting changes in patient therapy management for one third to two thirds of patients who were undergoing the ${ }^{68} \mathrm{Ga}$ imaging. These therapy changes were due to a variety of reasons, including discovery of cancers of unknown primary leading to surgery, existence of additional metastatic disease, and change in eligibility for peptide receptor radionuclide therapy.

This growing body of work was presented at the first Theranostics World Congress in Germany in 2011, bringing together nearly 400 members of the nuclear medicine community to discuss their work with ${ }^{68} \mathrm{Ga}$ and other diagnostic-therapeutic pairs. Many of the referenced papers in this article were first presented at the Congress. The Congress continued to drive interest, and shortly thereafter the first U.S. Investigational New Drug Applications were filed with the Food and Drug Administration for ${ }^{68} \mathrm{Ga}$-DOTATATE and ${ }^{68} \mathrm{Ga}$-DOTATOC. Results from those trials and additional positive trials have been used to support a New Drug Application to the Food and Drug Administration for ${ }^{68} \mathrm{Ga}$-DOTATATE, which was approved in June 2016 for the imaging of NETs. As Graham et al. mention, the University of Iowa will soon be submitting a New Drug Application with the Food and Drug Administration for ${ }^{68} \mathrm{Ga}$-DOTATOC.

A great deal has happened in the 23 y since the OctreoScan was approved. In addition to ${ }^{68} \mathrm{Ga}$-DOTATATE's approval, 4 new therapeutics have been approved by the Food and Drug Administration this decade for the treatment of NETs (sunitinib, everolimus, lanreotide, 
telotristat), with an additional application currently under review (lutathera). With the advent of low-cost ${ }^{68} \mathrm{Ga}$ generators, many research institutions now have the capability to do research and clinical trials using targeted therapy with the same peptide by changing out the isotopes for diagnostic or therapeutic use.

As a patient with a rare disease, it has been encouraging to see the increase in the development of new imaging and treatment options. Reviewing the Graham et al. article reminds the entire patient community just how many researchers from around the world are vested in improving patient outcomes and quality of life. Although it took 22 y to bring forward a new approval for an imaging agent for NETs, there is no reason to wait an additional $22 \mathrm{y}$ for the next agent. DOTATOC and other targeted peptides will give clinicians new tools to work with in the management of NET patients. More tools will continue to increase access for patients to undergo diagnostic imaging and improved outcomes. I concur with the authors' closing statement that ${ }^{68} \mathrm{Ga}$-DOTATOC "appears to be an excellent imaging agent to assess patients with known NET and frequently leads to a change in management."

\section{REFERENCES}

1. Graham MM, Gu X, Ginader T, Breheny P, Sunderland JJ. ${ }^{68} \mathrm{Ga}$-DOTATOC imaging of neuroendocrine tumors: a systematic review and meta-analysis. J Nucl Med. 2017;58:1452-1458.

2. Dasari A, Shen C, Halperin D, et al. Trends in the incidence, prevalence, and survival outcomes in patients with neuroendocrine tumors in the United States. JAMA Oncol. April 27, 2017 [Epub ahead of print].

3. Balon HR, Brown TLY, Goldsmith SJ, et al. The SNM practice guideline for somatostatin receptor scintigraphy 2.0. J Nucl Med Technol. 2011;39:317324. 